\title{
BMJ Open Innovative STRoke Interactive Virtual thErapy (STRIVE) online platform for community-dwelling stroke survivors: a randomised controlled trial protocol
}

\author{
Liam Johnson, ${ }^{1,2,3}$ Marie-Louise Bird, ${ }^{4,5}$ Makii Muthalib, ${ }^{6,7,8}$ Wei-Peng Teo ${ }^{9}$
}

To cite: Johnson L, Bird M-L, Muthalib M, et al. Innovative STRoke Interactive Virtual thErapy (STRIVE) online platform for communitydwelling stroke survivors: a randomised controlled trial protocol. BMJ Open 2018;8:e018388. doi:10.1136/ bmjopen-2017-018388

- Prepublication history for this paper is available online. To view these files, please visit the journal online (http://dx.doi. org/10.1136/bmjopen-2017018388).

Received 26 June 2017 Revised 24 October 2017 Accepted 27 November 2017

CrossMark

For numbered affiliations see end of article.

Correspondence to

Dr Wei-Peng Teo;

weipeng.teo@deakin.edu.au

\section{ABSTRACT}

Introduction The STRoke Interactive Virtual thErapy (STRIVE) intervention provides community-dwelling stroke survivors access to individualised, remotely supervised progressive exercise training via an online platform. This trial aims to determine the clinical efficacy of the STRIVE intervention and its effect on brain activity in communitydwelling stroke survivors.

Methods and analysis In a multisite, assessor-blinded randomised controlled trial, 60 stroke survivors $>3$ months poststroke with mild-to-moderate upper extremity impairment will be recruited and equally randomised by location (Melbourne, Victoria or Launceston, Tasmania) to receive 8 weeks of virtual therapy (VT) at a local exercise training facility or usual care. Participants allocated to VT will perform 3-5 upper limb exercises individualised to their impairment severity and preference, while participants allocated to usual care will be asked to maintain their usual daily activities. The primary outcome measures will be upper limb motor function and impairment, which will be assessed using the Action Research Arm Test and Upper Extremity Fugl-Meyer, respectively. Secondary outcome measures include upper extremity function and spasticity, as measured by the box and block test and Modified AshworthScale, respectively, and task-related changes in bilateral sensorimotor cortex haemodynamics during hand reaching and wrist extension movements as measured by functional near-infrared spectroscopy. Quality of life will be measured using the Euro-Quality of Life-5 Dimension-5 Level Scale, and the Motor Activity Log-28 will be used to measure use of the hemiparetic arm. All measures will be assessed at baseline and immediately postintervention.

Ethics and dissemination The study was approved by the Deakin University Human Research Ethics Committee in May 2017 (No. 2017-087). The results will be disseminated in peer-reviewed journals and presented at major international stroke meetings.

Trial registration number ACTRN12617000745347; Preresults.

\section{INTRODUCTION}

Stroke is one of the leading causes of adult disability in Western countries, ${ }^{1}$ and for many stroke survivors, upper extremity (UE) paresis makes performing activities of daily

\section{Strengths and limitations of this study}

- Community-based access allows for greater community engagement and real-world translatability than in a controlled laboratory environment.

- The STRoke Interactive Virtual thErapy (STRIVE) trial allows experimenters to remotely access and control virtual therapy exercise programmes based on the participant's weekly performance data.

- Clinical and neurophysiological measures will determine functional and associated cortical changes in response to virtual therapy poststroke.

- While STRIVE is a community-based intervention, the trial is only accessible to participants that are mobile and can travel to the trial sites.

living (ADLs) difficult. Up to $60 \%$ of community-dwelling stroke survivors live with severe motor impairments of the shoulders, elbows and/or wrists that significantly impacts their functional capacity and quality of life. ${ }^{2}$ Improved UE function is considered a rehabilitation priority after stroke, ${ }^{3}$ yet optimal recovery of arm function is poor. ${ }^{24}$ A large majority of stroke survivors experience a lack of support and access to rehabilitative services once they are discharged into the community, ${ }^{56}$ which can compromise their recovery. While most recovery occurs in the first weeks to months after stroke, improvements in function can still be experienced beyond this period. ${ }^{7}$

The use of virtual reality as a therapy, which is characterised by the participant being immersed in, and interacting with, a computer-generated environment, ${ }^{8}$ is emerging as an efficacious treatment for UE impairment after stroke. ${ }^{910}$ Online virtual therapy (VT) systems can provide the fundamental elements needed for motor skill development; they can be individually tailored, involve many task-specific repetitions that are increasingly 


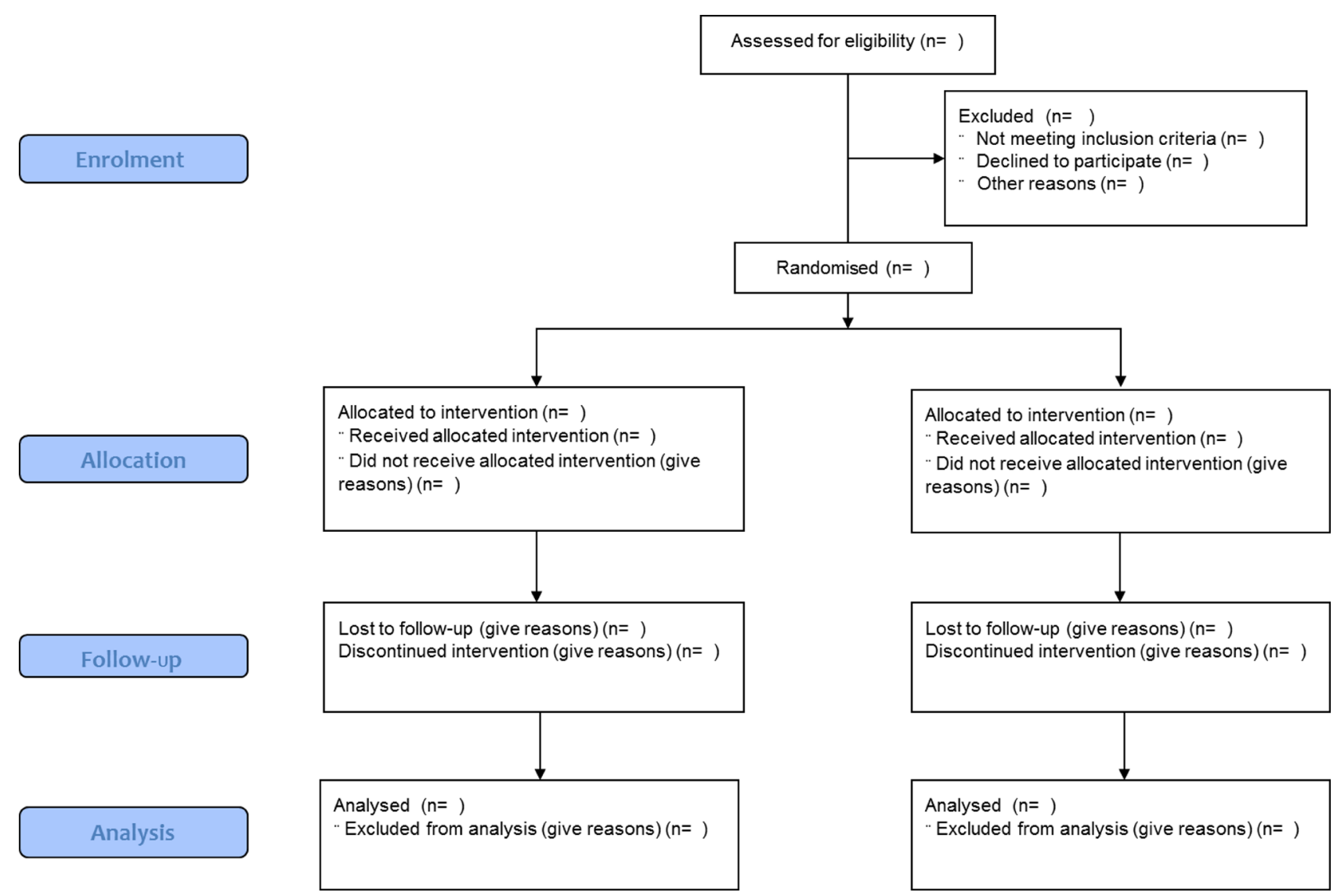

Figure 1 The CONSORT 2010 flow diagram for the study. CONSORT, Consolidated Standards of Reporting Trials.

challenging in response to participant improvement and feedback can be embedded in the system. The enriched environment offered by VT is thought to be effective in training problem solving and functional task performance ${ }^{11}$ and can potentially increase participant engagement compared with non-VT rehabilitation platforms. ${ }^{12}$

Online VT systems have the potential to address the lack of community-based rehabilitation support experienced by stroke survivors by being affordable, accessible, user-friendly and importantly, have the ability to remotely monitor rehabilitation progress. VT systems, such as the Jintronix Rehabilitation System (Montreal, Canada) to be used in this study, can be administered affordably through commercially available products that include motion capture capabilities (eg, Microsoft Xbox Kinect V.2) and personal computers. ${ }^{13}$ Online VT systems can be easily implemented at a local community centre, which would enable patients with stroke to receive specialised treatment and monitoring remotely. Online VT platforms have been shown to be user-friendly and motivating, ${ }^{14}$ including interfaces that are engaging and easy to interact with, and software that can be run on any personal computer/device. In a Cochrane review, Laver $e t$ at reported low-quality evidence suggesting VT is a more effective approach to improve arm function after stroke compared with conventional therapy. ${ }^{9}$ A recent multiple systematic review, including 10 randomised controlled trials and four systematic reviews, found VT therapy to be similar to standard rehabilitation for treatment of UE impairment and disabilities. ${ }^{15}$

To understand the effects of VT on cerebral activity in stroke rehabilitation, neuroimaging techniques such as functional MRI (fMRI) have been used previously to determine cortical reorganisation postrehabilitation. ${ }^{16}$ While fMRI is considered the gold-standard measure in neuroimaging, these techniques may be limited as they only allow for small movements to occur within the scanner that are very different from activities of daily living (ADLs). In this sense, functional near-infrared spectroscopy (fNIRS) may be a more suitable neuroimaging technique as it is able to measure changes in cerebral haemodynamic responses (ie, changes in oxyhaemoglobin and deoxyhaemoglobin $\left(\mathrm{HbO}_{2}\right.$ and $\left.\left.\mathrm{HHb}\right)\right)$ in response to larger body and head movements that mimic ADLs. Previous studies have also established that cerebral haemodynamic measures from fNIRS are highly comparable with blood oxygenlevel dependent signals from fMRI, ${ }^{17} 18$ which makes it a suitable surrogate to measure changes in brain activity following VT rehabilitation in people with stroke.

Given the advantages of increased accessibility to specialised treatment and monitoring that is afforded by VT, we aim to determine if an online VT system can provide efficacious UE rehabilitation for community-dwelling stroke survivors. We have chosen to focus our intervention on UE function as impaired arm function is highly common after stroke, ${ }^{2}$ which profoundly impacts the capacity to 
Capture as many stars as you can in the allotted time. Watch the example below.

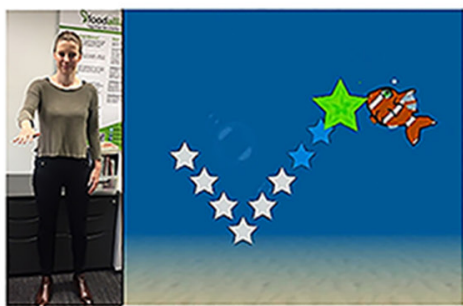

Reach and clap your hands over the balloons to pop them.

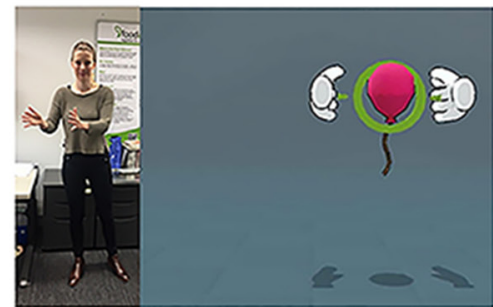

Reach with your hands to touch the green and blue balls and avoid the red balls.

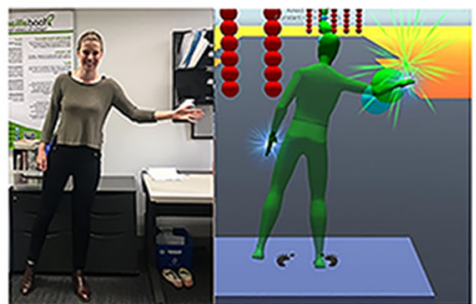

Get as many apples in to the pipes as you can! Watch the example below.

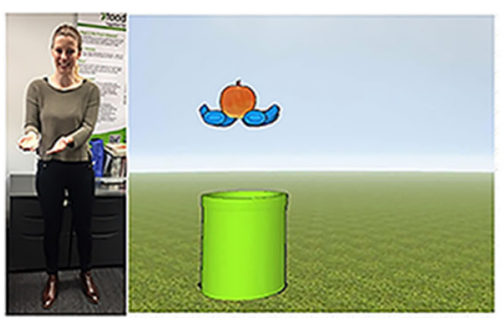

Figure 2 Examples of VT therapy games that target UE mobility of the shoulders, elbows and wrists. UE, upper extremity; VT, Virtual therapy.

perform ADLs ${ }^{19}$ and only a small number of stroke survivors experience complete functional recovery of the UE. ${ }^{20}$

\section{METHODS AND ANALYSIS \\ Study design}

This is a multisite, assessor-blinded randomised controlled trial to be conducted in two Australian centres (Melbourne, Victoria and Launceston, Tasmania). The Consolidated Standards of Reporting Trials (CONSORT) statement (figure 1) has been used to guide trial design. ${ }^{21}$ The trial will be reported according to the Standard Protocol Items: Recommendations for Interventional Trials (SPIRIT) guidelines ${ }^{22}$ and the Template for Intervention Description and Replication. ${ }^{23}$

\section{Participants}

Community-dwelling stroke survivors will be recruited to participate from the Melbourne metropolitan area, Victoria, and from Launceston, Tasmania. The study will be advertised on the Australian Stroke Foundation and Heart Foundation websites, through social media (eg, twitter and facebook), in local newspapers and at local community centres.

Potential participants will initiate contact with the research team by responding to the study advertisements by phone or email. The researchers will respond in kind and provide information about the project, and then send an electronic or paper copy of the plain language statement to the participant. All potential participants will then attend an initial screening session at Deakin University, Burwood, Melbourne or the University of Tasmania, Launceston, to determine their eligibility to participate according to the inclusion/exclusion criteria. Participants will be included if they are (1) above the age of 18 years, (2) community-dwelling stroke survivors,
(3) with mild-to-moderate UE impairments (Fugl-Meyer Upper Extremity (UE-FM) Score 25-45), (4) no cognitive impairments (Mini Mental State Examination Score $>24$ ), (5) at least 3 months poststroke, (6) can demonstrate an observable motor-evoked potential from the resting paretic hand, of at least $0.05 \mathrm{mV}$, in 5 out 10 trials during transcranial magnetic stimulation and (7) able to read English. Participants will be excluded from the study if they have (1) other neurological conditions (eg, Parkinson's disease or dementias), (2) no observable movements in shoulders or elbows and (3) upper extremity joint pain that would limit their ability to complete the trial.

If eligible, the principal investigator will provide the individual with a consent form to read and sign. The individual will be given the opportunity to ask the principal investigator any questions about the study and their potential participation in it. On providing signed informed consent, all individuals will be scheduled for baseline and postintervention testing at Deakin University Physical Activity and Nutrition research laboratory (Burwood, Melbourne, Australia) or the University of Tasmania Exercise Physiology Clinic (Launceston, Tasmania, Australia).

\section{Randomisation and blinding}

Following study enrolment and completion of the baseline testing, participants will be randomised into a VT intervention or usual care (control; UC) group via a computerised random sequence generator and using a block randomisation design (according to location) and stratified by gender.

\section{Location and setting}

The participants randomised to the VT group will undertake training at the High Street Road Uniting Church (for the Melbourne-based participants) or the University 
of Tasmania Exercise Physiology Clinic, Launceston (for the Tasmanian-based participants). A Microsoft Xbox Kinect V.2 (Microsoft, Redmond, Washington, USA) and a laptop will be placed at each training location. All participants will be instructed to complete a daily physical activity diary throughout the study.

An exercise physiologist with at least 2 years of experience working with people with neurological impairments and/or brain injuries will be located at each training site to provide technical support on the VT system and document any adverse events (AEs) .

\section{Intervention}

The participants in the VT group will attend their local exercise training facility twice weekly, with each session separated by at least 24 hours, for eight consecutive weeks to complete the VT training. Each VT session will last approximately $45-60 \mathrm{~min}$, which will equate to approximately $12-16$ hours of total therapy time across the duration of the study. This total amount of therapy is consistent with previous research as necessary to elicit improvements in motor function. ${ }^{9}$ Figure 2 contains examples of the UE exercises that will be used in this study.

The Jintronix Rehabilitation System (Montreal, Canada) to be used in this study will assess the participants active range of motion (ROM) of the shoulders, elbows and wrists, which will be used to establish the initial ROM of the exercises. The prescription of UE exercises will be determined by the researcher at the initial session. In the initial training session, the participants will be asked to provide feedback on their preference and capacity to perform the prescribed exercises. If the participants report experiencing difficulties with the intensity and type of exercises prescribed, an alternative exercise, that still targets the intended muscle group and action, will be selected. The exercise type and intensity will be selected and tailored to target the individual's UE impairment, which will be based on the individual's baseline function and active ROM of the shoulder, elbow and wrist. The Jintronix Rehabilitation System records each participant's performance (ie, number of task repetitions, time in therapy for each exercise and task performance accuracy) for each session completed, and the training intensity for each participant will be modified according to their performance during the previous week. An advantage of the Jintronix Rehabilitation System is the capability of the system to adjust the training stimulus by changing the number of repetitions performed, and the ROM that the exercises are performed in, as the participant adapts over the duration of the intervention.

Any participants who are wheelchair users or have difficulty standing for long periods may perform their exercises while seated, with the Jintronix system capable of detecting UE movement in either a standing or seated position. Monitoring of the training programme and modification in exercise prescription will be performed remotely by an exercise physiologist at Deakin University at the end of each week via the clinician's interface module of the Jintronix software. They will not have any direct contact with the participants, but will tailor each participant's VT training in response to their adaptation to the programme. Progression of each exercise will be based on achievement of at least $80 \%$ success (ie, the participant is able to hit at least 8 out of 10 targets in a game) in exercise performance over two consecutive training sessions. The difficulty of the exercise will be progressed by $10 \%$, which may consist of, but not limited to, more targets to hit, need for greater active ROM to successfully perform the task or quicker movement time to target. If a participant achieves a score of $<50 \%$ in two consecutive exercise sessions for a particular exercise, the level of difficulty for that particular exercise will be reduced by $10 \%$.

\section{Control}

$\mathrm{UC}$, in the context of this study, is defined as maintaining the level of physical activity similar to that prior to enrolment into the trial. All participants allocated to the UC control group will be requested to maintain their daily routine and any existing therapy, and to report any change in the dose of their therapy. The UC group participants will only be required to attend the testing sessions (outlined below) at baseline and after 8 weeks.

\section{Outcome measures}

Table 1 shows the standard protocol items and outcome measures as recommended by the SPIRIT guidelines for interventional trials. A change in the Action Research Arm Test (ARAT) score and the UE-FM scale from preintervention to postintervention will be joint primary outcomes in this study.

The ARAT is a measure of arm motor function that consists of 19 items grouped into four subscales: grasp, grip, pinch and gross movement. ${ }^{24}$ It uses ordinal scoring, with each item scored as either a 0 ('cannot perform any part of the test'), 1 ('performs the test partially'), 2 ('completes the test, but takes abnormally long time') or 3 ('performs the test normally'), with a lower score indicating reduced UE function. ${ }^{24}$ The ARAT has established reliability ${ }^{2526}$ and validity, is responsive to change ${ }^{27}$ and has good concurrent validity compared with other measures used to assess arm motor function. ${ }^{28}$

The UE-FM assessment is a stroke-specific functional performance measure that includes 33 questions (maximum score of 66) designed to assess muscle strength, reflexive activity and motor control. ${ }^{29}$ Each item is scored as either a 0 ('unable to perform'), 1 ('able to perform in part') or 2 ('able to perform'), with a lower score indicating reduced UE function. ${ }^{29} 30$ The UE-FM is a reliable and valid assessment of UE motor function, ${ }^{31}$ that is sensitive to change. ${ }^{32}$

Secondary outcome measures include the box and block test $(B B T)$, which provides a measure of arm and hand function, and the Modified Ashworth Scale (MAS) score, which measures upper and lower limb spasticity. The Motor Activity Log-28 (MAL-28) and Euro-Quality of Life-5 
Table 1 Standard Protocol Items: Recommendations for Interventional Trials ${ }^{22}$

\begin{tabular}{|c|c|c|c|}
\hline & \multicolumn{3}{|c|}{ Study period } \\
\hline & Enrolment & Preallocation & Postallocation \\
\hline Time point & $-t_{1}$ & $t_{1}$ & $t_{2}$ \\
\hline \multicolumn{4}{|l|}{ Enrolment } \\
\hline Eligibility screen & $x$ & & \\
\hline Informed consent & $x$ & & \\
\hline Randomisation & & $\mathrm{x}$ & \\
\hline \multicolumn{4}{|l|}{ Intervention } \\
\hline \multicolumn{4}{|l|}{ Virtual therapy } \\
\hline \multicolumn{4}{|l|}{ Usual care } \\
\hline \multicolumn{4}{|l|}{ Assessment } \\
\hline Action Research Arm Test & & $\mathrm{x}$ & $x$ \\
\hline Fugl-Meyer Upper ExtremityAssessment & & $\mathrm{x}$ & $x$ \\
\hline Modified Ashworth Scale & & $x$ & $x$ \\
\hline Mini-Mental State Examination & & $\mathrm{x}$ & $x$ \\
\hline Functional near-infrared spectroscopy & & $\mathrm{x}$ & $x$ \\
\hline EQ-5D-5L & & $\mathrm{x}$ & $\mathrm{x}$ \\
\hline Box and block test & & $x$ & $x$ \\
\hline Motor activity log-28 & & $\mathrm{x}$ & $x$ \\
\hline
\end{tabular}

EQ-5D-5L, Euro-Quality of life-5 Dimension-5 Level.

Dimension-5 Level (EQ-5D-5L) Scale will be used to document the daily use of the stroke-affected arm and how VT may impact on quality of life (QoL), respectively. fNIRS will be used to measure task-related changes in haemodynamic responses of the bilateral primary sensorimotor cortices (SMCs).

The BBT is commonly used to measure unilateral gross motor dexterity of the arm and hand poststroke. ${ }^{33}$ During the BBT, participants will be seated at a table, facing a rectangular box that is partitioned into two square compartments of equal dimension. One hundred and fifty, $2.5 \mathrm{~cm}$, coloured, wooden blocks are placed in one compartment, and the participant will be instructed to move as many blocks as possible, one at a time, to the other compartment in $60 \mathrm{~s}$. The BBT is scored by counting the number of blocks carried over the partition from one compartment to the other during the $60 \mathrm{~s}$ trial period. In order to score a point, the participant's hand must cross over the partition, and blocks that drop or bounce out of the second compartment onto the table or floor are still recorded as a point. Should multiple blocks be carried over at the same time, only a single point will be recorded. Higher scores on the test indicate better gross motor dexterity. Previous studies using the BBT in people with stroke have shown excellent test-retest reliability $(\mathrm{ICC}=0.96)$ and inter-rater reliability $(\mathrm{ICC}=0.99) .{ }^{34}$

The MAS is commonly used to measure the intensity of muscle spasticity experienced poststroke in the upper and lower extremities. ${ }^{35}$ Relevant muscle groups are passively moved about a joint and scored on a 0 ('no increase in tone') to 4 ('affected part(s) rigid in flexion or extension') scale, with a lower score indicating a lower resistance to movement (ie, reduced spasticity). The MAS has excellent inter-rater and intrarater reliability ${ }^{36}$ and convergent validity with motor performance $e^{37} 38$ and neurophysiological measures. ${ }^{37}$

The MAL-28 is a stroke-specific self-report questionnaire that quantifies UE performance in real world activities. ${ }^{39}$ The participant will be asked to rate their arm function each week according to two subscales: the Quality of Movement (QOM) scale and the Amount of Use (AOU) scale. Each scale is rated on a scale of 0 ('the weaker arm was not used at all for that activity' on the QOM scale, and 'did not use my weaker arm' on the AOU scale) to 5 ('the ability to use the weaker arm for that activity was as good as before the stroke' on the QOM and 'used my weaker arm as often as before the stroke' on the AOU scale) ${ }^{39}$ Scores on each scale are calculated as the mean of the scored items attempted with the affected arm, with a higher average score indicative of better QOM and more use of the weaker arm.

The EQ-5D-5L provides an assessment of health-related QoL after stroke across five dimensions: mobility, self-care, usual activities, pain/discomfort and anxiety/depression. ${ }^{40}$ Each dimension is divided into 5 levels of perceived problems: no problems (level 1), slight problems (level 2), moderate problems (level 3), severe problems (level 4) and extreme problems (level 5). The participant will be asked to indicate their health state by ticking the box corresponding to the most appropriate statement in each of the five dimensions. Once all five dimensions have been scored, the digits for the five dimensions are combined 
into a 5-digit number that describes the patient's health state. Lower scores indicate a higher QoL. The EQ-5D-5L is a valid measure of health-related QoL after stroke, ${ }^{40}$ with acceptable responsiveness. ${ }^{41}$

fNIRS is a non-invasive and portable optical neuroimaging technique that can measure task-related changes in cortical haemodynamics (ie, an indirect measure of cortical activation) in stroke survivors, ${ }^{42}$ but without the large costs and non-portability of fMRI assessments. fNIRS has previously been used to measure changes in prefrontal cortical activation during a virtual reality movement task in healthy individuals. ${ }^{43}$ In this study, fNIRS will be used to measure bilateral SMC activation during functional seated hand reaching and wrist extension movement tasks of the paretic and non-paretic arm. The assessment of these motor task-related bilateral SMC activation changes after the STRIVE intervention will provide a cortical correlate of the change in motor function, ${ }^{44}$ as quantified using the Kinect V.2 with the Macoki software (NaturalPad, Montpellier, France). ${ }^{45}$

\section{Compliance and safety monitoring}

The VT system provides information on usage time during each training session, which will be remotely monitored as a measure of exercise compliance. In addition, the attending exercise physiologist will record the participant's attendance. Should a participant miss a training session, they will be contacted by the attending exercise physiologist and the reason for their absence will be documented. If more than one training session is missed, a make-up session will be scheduled. The attending exercise physiologist will also document and handle any AEs (ie, muscle or joint pain, syncope, falls and injuries) or serious adverse events (SAEs, ie, any incident requiring hospitalisation). ${ }^{46}$ The study is voluntary and participants can discontinue the study on request at any time, or if there is a change in health status (ie, worsening of function or overall health) or in the event of an AE or SAE. All AEs and SAEs experienced by the study participants during the trial will be reported immediately to the principal investigator and the Deakin University Human Research Ethics Committee. Should any participant suffer from any AE or SAE because of this trial, ancillary support and/or post-trial care will be provided.

\section{Sample size estimation}

To successfully detect a minimal clinically important difference in the UE-FM score of $6.4 \%-11 \%$ (an increase of 4.25-7.25 points out of 66), ${ }^{47} 30$ participants in each group (VT and UC) will be sufficient to provide $80 \%$ power $(95 \% \mathrm{CI})$ and account for a $30 \%$ dropout rate.

\section{Statistical analysis plan}

Mixed factorial analysis of variance will be used to determine the difference in clinical and neurophysiological measures across GROUP (VT vs Control) and TIME (PREINTERVENTION vs POSTINTERVENTION). Bonferroni post hoc t-tests for multiple comparisons will be used to determine where significance has occurred. Significance will be set at $\mathrm{P}<0.05$.

\section{ETHICS AND DISSEMINATION}

The primary results regarding the efficacy of the STRIVE intervention to improve UE function and spasticity, and QoL poststroke will be published in a peer-reviewed journal and presented at an international conference. Separate manuscripts will be written for the neurobiological secondary outcome measures. These will also be submitted for publication in peer-reviewed journals and presented at appropriate conferences. A summary of the main findings of the study will be made available to the participants involved in the study, and the Heart Foundation who have funded this study. All personal data of potential and enrolled participants will be deidentified and stored in a locked filing cabinet (physical documents) and password-protected work computer (participant's results and relevant softcopy documents) for a minimum of 5 years with the principal investigator. Should there be any changes to the protocol (ie, eligibility criteria, outcomes, analyses or project extension), an ethics amendment form will be submitted to DUHREC for consideration and approval.

Following completion of the study and publication of the findings, all data will be deposited in the Deakin University data repository and made freely available on request to the corresponding author.

\section{DISCUSSION}

Despite adequate inpatient rehabilitation support, and evidence that indicates functional improvements can be made many months poststroke, ${ }^{3}$ optimal recovery of UE function is poor. ${ }^{2}$ While stroke survivors are strongly advised to continue with physical rehabilitation on discharge into the community, they often face a lack of support and access to rehabilitative services. The STRIVE study will investigate the effectiveness of VT therapy compared with UC in reducing UE impairment after stroke.

The STRIVE intervention is designed to offer stroke survivors individualised therapy, featuring highly repetitive task-specific movements, prescriptive feedback and progression within an enriched environment. The Jintronix Rehabilitation System is highly modifiable and adaptive to the abilities of the user-a critical feature given the expected variability in the stroke cohort.

The clinically oriented primary (ie, ARAT and UE-FM) and secondary (ie, BBT and MAS) outcomes of the STRIVE study will assess the efficacy of VT rehabilitation in modifying arm function and spasticity. The MAL-28 and EQ-5D-5L assessments will indicate the impact of the STRIVE intervention on the functional ability and QoL of the participants. Furthermore, the secondary outcome of fNIRS neuroimaging will provide an insight into VT-mediated neuroplastic changes in 
the bilateral SMC that will aid in furthering our understanding of the central adaptations implicated in UE motor function recovery.

The strengths of the STRIVE study include the tailored approach to the initial therapy prescription, and also the responsiveness of the intervention to meet the changing abilities of the user over the duration of the intervention. The attendance of an exercise physiologist at the therapy sessions aims to address a common complaint by users of technology-based therapy, that is, the challenge in setting up the technology and problem-solving technical issues. It also is a pragmatic approach to ensure the safety of the participants. Furthermore, the Microsoft Xbox Kinect V.2 is a commercially-available device that can be set-up at a very low cost and is compatible with devices commonly found in a local community centre, making it a highly accessible device.

The STRIVE study will afford community-dwelling stroke survivors the opportunity for supported access to rehabilitation. Our findings will assist clinicians in the prescription of efficacious rehabilitation for patients using a low-cost, online VT platform that can be implemented into a community setting and integrated into the user's daily life. This may lower the burden of disease by ensuring stroke survivors can receive specialised treatment and monitoring from within their local community beyond the initial phase of stroke recovery, which may improve rehabilitation compliance and outcomes in the long term.

\section{Author affiliations}

${ }^{1}$ Stroke Division, The Florey Institute of Neuroscience and Mental Health, University of Melbourne, Heidelberg, Victoria, Australia

${ }^{2}$ Faculty of Health Sciences, Australian Catholic University, Melbourne, Australia ${ }^{3}$ Institute of Sport, Exercise and Active Living (ISEAL), Victoria University, Melbourne, Australia

${ }^{4}$ Faculty of Health, School of Health Sciences, University of Tasmania, Launceston, Tasmania, Australia

${ }^{5}$ Department of Physical Therapy, University of British Columbia and the

Rehabilitation Research Program, GF Strong Rehabilitation Centre, University of British Columbia, Vancouver, Canada

${ }^{6}$ EuroMov, University of Montpellier, Montpellier, France

${ }^{7}$ Cognitive Neuroscience Unit, School of Psychology, Deakin University, Burwood, Victoria, Australia

${ }^{8}$ SilverLine Research Services, Brisbane, Australia

${ }^{9}$ Institute for Physical Activity and Nutrition (IPAN), School of Exercise and Nutrition

Sciences, Deakin University, Burwood, Victoria, Australia

Acknowledgements We would like to thank Ms Jessica Freeman and Ms Karima Bakhti for their assistance in developing the study. Ms Freeman has provided consent for her image to be published in figure 2 .

Contributors W-PT, LJ, MM and M-LB: were involved in conception and trial design. LJ: wrote the first draft of this manuscript. W-PT, LJ, MM and M-LB: were involved in critical revision of the article for important intellectual content. All the authors were involved in final approval of the article.

Funding This work was supported by a Heart Foundation Vanguard Grant (101350). W-PT is supported by an Alfred Deakin Postdoctoral Research Fellowship. MM was supported by Silverline research services.

Competing interests None declared.

Ethics approval Deakin University Human Research Ethics Committee (DUHREC, no. 2017-087).
Provenance and peer review Not commissioned; externally peer reviewed.

Open Access This is an Open Access article distributed in accordance with the Creative Commons Attribution Non Commercial (CC BY-NC 4.0) license, which permits others to distribute, remix, adapt, build upon this work non-commercially, and license their derivative works on different terms, provided the original work is properly cited and the use is non-commercial. See: http://creativecommons.org/ licenses/by-nc/4.0/

(C) Article author(s) (or their employer(s) unless otherwise stated in the text of the article) 2018. All rights reserved. No commercial use is permitted unless otherwise expressly granted.

\section{REFERENCES}

1. Feigin VL, Krishnamurthi RV, Parmar $P$, et al. Update on the global burden of ischemic and hemorrhagic stroke in 1990-2013: The GBD 2013 study. Neuroepidemiology 2015;45:161-76.

2. Kwakkel G, Kollen BJ, van der Grond J, et al. Probability of regaining dexterity in the flaccid upper limb: impact of severity of paresis and time since onset in acute stroke. Stroke 2003;34:2181-6.

3. Barker RN, Gill TJ, Brauer SG. Factors contributing to upper limb recovery after stroke: a survey of stroke survivors in Queensland Australia. Disabil Rehabil 2007;29:981-9.

4. Brauer SG, Hayward KS, Carson RG, et al. The efficacy of SMART Arm training early after stroke for stroke survivors with severe upper limb disability: a protocol for a randomised controlled trial. BMC Neurol 2013;13:71.

5. Pollack MR, Disler PB. Rehabilitation of patients after stroke. Med J Aust 2002;177:452-6.

6. Stroke Foundation. National stroke audit - rehabilitation services report. Melbourne, Australia: Stroke Foundation, 2016.

7. Teasell RW, Murie Fernandez M, Mclntyre A, et al. Rethinking the continuum of stroke rehabilitation. Arch Phys Med Rehabil 2014;95:595-6.

8. Weiss PL, Kizony R, Feintuch $U$, et al. Virtual reality in neurorehabilitation. In: Selzer M, Cohen L, Gage F, Clarke S, et al. eds. Textbook of neural repair and rehabilitation: Cambridge University Press, 2006:182-97.

9. Laver KE, George S, Thomas S, et al. Virtual reality for stroke rehabilitation. Cochrane Database Syst Rev 2015:CD008349.

10. Teo WP, Muthalib M, Yamin S, et al. Does a combination of virtual reality, neuromodulation and neuroimaging provide a comprehensive platform for neurorehabilitation? - a narrative review of the literature. Front Hum Neurosci 2016;10:284.

11. Risedal A, Mattsson B, Dahlqvist $P$, et al. Environmental influences on functional outcome after a cortical infarct in the rat. Brain Res Bull 2002;58:315-21.

12. Laut J, Cappa F, Nov O, et al. Increasing patient engagement in rehabilitation exercises using computer-based citizen science. PLoS One 2015;10:e0117013.

13. Bird ML, Cannell J, Callisaya ML, et al. "FIND Technology": investigating the feasibility, efficacy and safety of controller-free interactive digital rehabilitation technology in an inpatient stroke population: study protocol for a randomized controlled trial. Trials 2016;17:203.

14. Lloréns R, Noé E, Colomer C, et al. Effectiveness, usability, and cost-benefit of a virtual reality-based telerehabilitation program for balance recovery after stroke: a randomized controlled trial. Arch Phys Med Rehabil 2015;96:418-25.

15. Hatem SM, Saussez G, Della Faille M, et al. Rehabilitation of motor function after stroke: a multiple systematic review focused on techniques to stimulate upper extremity recovery. Front Hum Neurosci 2016;10:442.

16. You SH, Jang SH, Kim YH, et al. Virtual reality-induced cortical reorganization and associated locomotor recovery in chronic stroke: an experimenter-blind randomized study. Stroke 2005;36:1166-71.

17. Anwar AR, Muthalib M, Perrey S, et al. Effective connectivity of cortical sensorimotor networks during finger movement tasks: a simultaneous fNIRS, fMRI, EEG study. Brain Topogr 2016;29:645-60.

18. Muthalib M, Anwar AR, Perrey S, et al. Multimodal integration of fNIRS, fMRI and EEG neuroimaging. Clin Neurophysiol 2013;124:2060-2.

19. Pollock A, Farmer SE, Brady MC, et al. Interventions for improving upper limb function after stroke. Cochrane Database Syst Rev 2014:CD010820.

20. Nakayama H, Jørgensen HS, Raaschou HO, et al. Recovery of upper extremity function in stroke patients: the Copenhagen stroke study. Arch Phys Med Rehabil 1994;75:394-8. 
21. Moher D, Schulz KF, Altman DG. The CONSORT statement: revised recommendations for improving the quality of reports of parallelgroup randomised trials. Lancet 2001;357:1191-4.

22. Chan AW, Tetzlaff JM, Altman DG, et al. SPIRIT 2013 statement: defining standard protocol items for clinical trials. Ann Intern Med 2013;158:200-7.

23. Hoffmann TC, Glasziou PP, Boutron I, et al. Better reporting of interventions: template for intervention description and replication (TIDieR) checklist and guide. BMJ 2014;348:g1687.

24. Lyle RC. A performance test for assessment of upper limb function in physical rehabilitation treatment and research. Int $J$ Rehabil Res 1981;4:483-92.

25. Hsieh CL, Hsueh IP, Chiang FM, et al. Inter-rater reliability and validity of the action research arm test in stroke patients. Age Ageing 1998;27:107-13.

26. Van der Lee JH, De Groot V, Beckerman $\mathrm{H}$, et al. The intra- and interrater reliability of the action research arm test: a practical test of upper extremity function in patients with stroke. Arch Phys Med Rehabil 2001;82:14-19.

27. Lang CE, Wagner JM, Dromerick AW, et al. Measurement of upperextremity function early after stroke: properties of the action research arm test. Arch Phys Med Rehabil 2006;87:1605-10.

28. Lin JH, Hsu MJ, Sheu CF, et al. Psychometric comparisons of 4 measures for assessing upper-extremity function in people with stroke. Phys Ther 2009;89:840-50.

29. Deakin A, Hill H, Pomeroy VM. Rough guide to the Fugl-Meyer assessment: upper limb segment. Physiotherapy 2003;89:751-63.

30. Singer B, Garcia-Vega J. The Fugl-Meyer upper extremity scale. J Physiother 2017;63:53.

31. Gladstone DJ, Danells CJ, Black SE. The Fugl-Meyer assessment of motor recovery after stroke: a critical review of its measurement properties. Neurorehabil Neural Repair 2002;16:232-40.

32. Hsieh YW, Wu CY, Lin KC, et al. Responsiveness and validity of three outcome measures of motor function after stroke rehabilitation. Stroke 2009;40:1386-91.

33. Desrosiers J, Bravo G, Hébert R, et al. Validation of the box and block test as a measure of dexterity of elderly people: reliability, validity, and norms studies. Arch Phys Med Rehabil 1994;75:751-5.

34. Platz T, Pinkowski $C$, van Wijck $F$, et al. Reliability and validity of arm function assessment with standardized guidelines for the Fugl-Meyer test, action research arm test and box and block test: a multicentre study. Clin Rehabil 2005;19:404-11.

35. Bohannon RW, Smith MB. Interrater reliability of a modified Ashworth scale of muscle spasticity. Phys Ther 1987;67:206-7.

36. Gregson JM, Leathley MJ, Moore AP, et al. Reliability of measurements of muscle tone and muscle power in stroke patients. Age Ageing 2000;29:223-8.

37. Katz RT, Rovai GP, Brait C, et al. Objective quantification of spastic hypertonia: correlation with clinical findings. Arch Phys Med Rehabil 1992;73:339-47.

38. Lin FM, Sabbahi M. Correlation of spasticity with hyperactive stretch reflexes and motor dysfunction in hemiplegia. Arch Phys Med Rehabil 1999;80:526-30.

39. Uswatte G, Taub E, Morris D, et al. The motor activity Log-28: assessing daily use of the hemiparetic arm after stroke. Neurology 2006;67:1189-94.

40. Golicki D, Niewada M, Buczek J, et al. Validity of EQ-5D-5L in stroke. Qual Life Res 2015;24:845-50.

41. Chen P, Lin KC, Liing RJ, et al. Validity, responsiveness, and minimal clinically important difference of EQ-5D-5L in stroke patients undergoing rehabilitation. Qual Life Res 2016;25:1585-96.

42. Mihara M, Miyai I. Review of functional near-infrared spectroscopy in neurorehabilitation. Neurophotonics 2016;3:031414.

43. Basso Moro S, Bisconti S, Muthalib M, et al. A semi-immersive virtual reality incremental swing balance task activates prefrontal cortex: a functional near-infrared spectroscopy study. Neuroimage 2014;85(Pt 1):451-60.

44. Lin PY, Chen JJ, Lin SI. The cortical control of cycling exercise in stroke patients: an fNIRS study. Hum Brain Mapp 2013;34:2381-90.

45. Bakhti K, Muthalib M, Perrey S, et al. fNIRS provides clues about the neural correlates of the learned non-use of the paretic arm after a stroke. Ann Phys Rehabil Med 2016;59:e77-e78.

46. Wallace S, Myles PS, Zeps N, et al. Serious adverse event reporting in investigator-initiated clinical trials. Med J Aust 2016;204:231-3.

47. Page SJ, Levine P, Hade E. Psychometric properties and administration of the wrist/hand subscales of the Fugl-Meyer Assessment in minimally impaired upper extremity hemiparesis in stroke. Arch Phys Med Rehabil 2012;93:2373-6. 